\title{
Cytoplasmic Inheritance in Paramecium: An Overview
}

\author{
Rajesh Wakchaure $^{1}$, Subha Ganguly ${ }^{2}$ and Praveen Kumar Praveen ${ }^{3}$ \\ ${ }^{1}$ Associate Professor, Department of Animal Genetics \& Breeding, \\ ${ }^{2}$ Associate Professor, Department of Veterinary Microbiology, \\ ${ }^{3}$ Assistant Professor, Department of Veterinary Public Health \& Epidemiology, ARAWALI \\ VETERINARY COLLEGE (Affiliated with Rajasthan University of Veterinary and Animal Sciences, \\ Bikaner), Rajasthan, India
}

*Corresponding Author: Dr. Subha Ganguly,

\begin{abstract}
In normal conditions, the phenotype of the progeny depends on the genes present in the nucleus and is called as chromosomal inheritance, where there is a simple association between the genes located on the chromosomes and observed phenotypes in the progeny. The male gametes and female gametes equally contribute to the phenotype of progeny and there is no differences observed with phenotypes with reciprocal crosses. In some exceptional cases, the phenotype is not dependent on the nuclear genes and is controlled by the genetic content present in the cytoplasm.
\end{abstract}

Keywords: Inheritance, Gamete, Paramecium

\section{INTRODUCTION}

The control of phenotype of the progeny by the non nucleus component present in the cytoplasm is called extrachromosomal inheritance or organellar inheritance or cytoplasmic inheritance. Extrachromosomal inheritance is distinct from the Maternal effect, where the phenotype of progeny depends on the mother's genotype and on nuclear gene products present in the cytoplasm of the egg. The genetic material present in the mitochondria or chloroplast are responsible for the extrachromosomal inheritance and hence called organellar inheritance since these organs are responsible for phenotype of the progeny. In few cases apart from mitochondria or chloroplast, the phenotype of the progeny depends on the extra-chromosomal particles present in the cytoplasm, hence also referred as cytoplasmic inheritance. In extrachromosomal inheritance, the reciprocal crosses between male and females will give different outcome. ${ }^{[1]}$

\section{Kappa particles in Paramecium}

The phenomenon of the killer trait was discovered in 1938 by Sonneborn ${ }^{[2]}$. He studied that Paramecium strains can be distinguished in "killer" and "sensitive" paramecia. Members of sensitive strain and members of a killer strain were mixed, the sensitive paramecia died within a few hours whereas the killer cells survived. Therefore the killer trait in Paramecium ${ }^{[3]}$ was recognized as a competitive benefit for killer cells. Kappa particles were identified as gram-negative bacteria ${ }^{[1]}$ and therefore had to be renamed ${ }^{[4]}$ using binominal nomenclature according to the Bacteriological Code. Kappa particles in Paramecium are other classical example for extrachromosomal inheritance, where the phenotype not only depends on the cytoplasm but also on the genetic material of the nucleus. ${ }^{[4]}$ The control of phenotype of the progeny by the non-nucleus component present in the cytoplasm is called extrachromosomal inheritance or organellar inheritance or cytoplasmic inheritance. The advanced research on extrachromosomal inheritance associate with human diseases will go a long way in genetic counseling. ${ }^{[5]}$

In Paramecium, small granular particles are present in the cytoplasm called as kappa particles. Kappa particles produce a protein called paramecin that kills the sensitive strain without kappa particles. Paramecin is the toxic substance produced by certain strains of Paramecium which kills the sensitive strains. The strain of Paramecium that produces paramecin is called Killer strain and hosts particles called Kappa particles which are responsible for paramecin production. Kappa particles require 
dominant allele $\mathrm{K}$ for its multiplication and genotypes KK and Kk can support Kappa particles, while genotype kk cannot maintain Kappa particles. ${ }^{[5]}$ Different results are observed when Killer strain is allowed to conjugate with sensitive strain depending on the duration of conjugation. In short conjugation, where only genetic material is exchanged without the cytoplasmic exchange between conjugates, both sensitive and killer strains are produced. In prolonged conjugation, it involves the exchange of both genetic and cytoplasmic content between conjugates. ${ }^{[6]}$

Paramecium reproduces both by sexual and asexual mode. During asexual reproduction, when conditions favour the cells undergo cell division while the kappa particles cannot divide at the same rate. Therefore the daughter cells receive less number of kappa particles and after few generations there can be complete loss of kappa particles. The strain with the kappa particles is called as killer/resistant strain and the other strain is called as sensitive strain. ${ }^{[7]}$ Gene responsible for producing kappa particles is present in homozygous or heterozygous dominant condition in killer strain while it is present in recessive form in sensitive strain. In this situation, strains with dominant alleles remain without any kappa particles. This proves the gene for kappa particles can only help in multiplication of existing kappa particles. During sexual reproduction, when conjugation period increases, the kappa particles move from killer strain to sensitive strain through conjugation tube converting sensitive strain to killer strain. ${ }^{[8]}$ The Kappa particles present in cytoplasm of killer strains enter the non-killer strains and convert it into killer type ,so all the progeny produced by exconjugates will have killer strains. ${ }^{[9,10]}$

Paramecium become killer strains when it receive Kappa particles and it become sensitive strains when it does not receive Kappa particles.

\section{Conclusion}

\section{Advantages:}

Extrachromosomal inheritance has some practical advantages in agriculture and can also be used in the prediction of diseases in humans for genetic counseling. In plants, cytoplasmic male sterility trait can be used for plant breeding. A recent clinical study involved congenital heart disease parents showed that the risk of getting heart disease in the progeny was higher if the mother is affected rather than the father. ${ }^{[5,6]}$

\section{REFERENCES}

[1] Hamilton, L.D. and Gettner, M.E. (1958). Fine structure of Kappa in Paramecium aurelia. The Journal of Biophysical and Biochemical Cytology, 4: 122-123.

[2] Sonneborn, T.M. (1938). Mating types in Paramecium aurelia: diverse conditions for mating in different stocks; occurrence, number and interrelations of the types. Proceedings of the National Academy of Sciences of the United States of America, 79: 411-434.

[3] Sonneborn, T.M. (1943): Gene and cytoplasm. I. The determination and inheritance of the killer character in variety 4 of Paramecium aurelia. Proceedings of the National Academy of Sciences of the United States of America, 29: 329-338.

[4] Preer, J.R. and Preer, L.B. (1982). Revival of names of protozoan endosymbionts and proposal of Holospora caryophila nom. nov. International Journal of Systematic Bacteriology, 32: 140141.

[5] Chandra Kala (2012). Extrachromosomal inheritance - Cytoplasmic influence of phenotype. Genetics, 15: 19-35.

[6] Ogar, Godwin Offumobi. (August 2014). A Written Term Paper Submitted in Partial fulfillments of the Requirements for the course (CBG 804) for the Degree of Master of Science (Cell Biology and Genetics), University of Lagos. Extrachromosomal inheritance in Paramecium. Supervisor: Dr. A. Khalid. https://www.academia.edu/24636373/EXTRACHROMOSOMAL_ INHERITANCE_IN_Paramecium

[7] Brown, C.H. (1950). Elimination of Kappa Particles from 'Killer' Strains of Paramecium aurelia by Treatment with Chloromycetin. Nature. 166 (4221): 527. doi:10.1038/166527A0

[8] Pond, F.R., Gibson, I., Lalucat, J. and Quackenbush, R.L. (1989-03-01). R-body-producing bacteria. Microbiological Reviews. 53 (1): 25-67. 
[9] Preer, L. B., Jurand, A., Preer Jr, J.R. and Rudman, B. M. (1972). The classes of kappa in Paramecium aurelia. Journal of Cell Science. 11 (2): 581-600.

[10] Preer Jr, J.R., Preer, L.B. and Jurand, A. (1974). Kappa and other endosymbionts in Paramecium aurelia. Bacteriological reviews. 38 (2): 113-163. 